\title{
A CONTRIBUTION TO THE THEORY OF THE POTENTIAL SWEEP METHOD: CHARGE TRANSFER REACTIONS WITH UNCOMPENSATED CELL RESISTANCE
}

\author{
N. R. de Tacconi, A. J. Calandra and A. J. Arvía \\ Instituto de Investigaciones Fisicoquimicas Teóricas y Aplicadas, División Electroquimica, Facultad \\ de Ciencias Exactas. Universidad Nacional de La Plata. La Plata. Argentina
}

(Received 5 June 1972; in revised form 22 January 1973)

\begin{abstract}
The effect of uncompensated cell resistance on current-potential curves obtained with the potential sweep method is presented for simple electrochemical reactions in conditions of a constant ionic activity and with the product entirely adsorbed on the electrode surface under either quasiequilibrium or Tafel conditions. First and second order processes are considered. Results obtained for the latter are referred to the electrochemical reduction of platinum oxide film in $1 N$ sulphuric acid at room temperature.
\end{abstract}

\section{INTRODUCTION}

The theory of the potential sweep method has been developed for electrochemical processes of different type, such as, Nernstian charge transfer with pyre diffusion control, for slow charge transfer, for dimerisation and polymerisation processes[1-15]. When a faradaic current flows through the working electrode, the cell resistance provokes the following distortion of the voltagram: (i) the current peak potentials are shifted toward larger values; (ii) the height of the current peaks is lowered, and (iii) the peak widths are broader. The magnitude of these effects increases with the cell resistance, the electrode area, the potential sweep rate and depolariser concentration. Therefore, the distortion of the voltagrams by uncompensated cell resistance and the dependence of this effect on potential sweep rate and depolariser concentration, indicate the convenience of establishing mathematical relationships describing the voltammetric currentpotential curves, in order to determine kinetic measurements aimed for mechanistic conclusions.

The effect of ohmic drop is of particular significance in three limiting cases: (i) when high potential sweep rates are used; (ii) when the reaction occurs in a system of relatively low conductance, as often happens when non-aqueous electrolyte solutions are studied, and (iii) when the reaction product yielded by the electrochemical reaction is a non-conducting species which accumulates on the electrode surface, as often occur in phenomena related to passivity.

The present paper is mainly devoted to study the possible effect of the uncompensated ohmic resistance on linear sweep voltammograms without, taking into account the double layer charging current, for simple electrochemical reactions in conditions where the activity of the ionic species is constant and the product is entirely adsorbed on the electrode surface.

\section{THEORETICAL DERIVATION}

Let us consider that a simple electron transfer reaction of the type:

$$
X^{-} \rightleftharpoons X+e
$$

occurs at the electrode, involving the formation of the adsorbed species $X$. It is assumed that reaction (1) in both directions is under activation control and species $X$, within the potential range to be considered, can be removed only by the ionisation process from the electrode surface. The concentration of the ionic species is considered as a constant from the outer Helmholtz plane throughout the bulk of the solution. Diffuse double layer effects are neglected. Then, the potential drop across the Helmholtz layer will differ from the potential applied between the working electrode and the reference electrode only by a constant term.

\section{Reaction (1) under quasi-equilibrium}

When an anodic potential sweep is applied to a system where reaction (1) occurs under quasiequilibrium conditions, the anodic faradaic current is given by:

$$
\begin{aligned}
i_{F}=k_{1}\left(\begin{array}{ll}
1 & \theta
\end{array}\right) a_{X^{-}} \exp & \left(\begin{array}{c}
\beta F V \\
R T
\end{array}\right) \\
& -k_{-1} \theta \operatorname{cxp}\left(-\frac{(1-\beta) F V}{R T}\right),
\end{aligned}
$$


where $k_{1}$ and $k_{-1}$ are the specific rate constants in the forward and backward directions when the potential difference, $V$, at the metal-solution interface is nill, and $\theta$ is the degree of surface coverage of the electrode by species $X$. When a linear potential sweep is applied both $V$ and $\theta$ certainly change with time. The expressions for the potential dependence of both the adsorption pseudo-capacitance and degree of surface coverage for reaction (1) under a linear potential sweep were derived, in the absence of ohmic polarisation, by different authors $[7-10,13]$. The conditions for this case are expressed as:

and

$$
i_{F} \ll k_{1} a_{X^{\prime}}(1-\theta) \exp \left(\frac{\beta F V}{R T}\right)
$$

$$
\left|i_{F}\right| \ll k_{-1} \theta \exp \left(-\frac{(1-\beta) F V}{R T}\right) .
$$

Taking into account (3) and (4) with (2):

$$
\begin{aligned}
k_{1}(1-\theta) a_{X^{-}} \exp & \left(\frac{\beta F V}{R T}\right) \\
& -k_{-1} \theta \exp \left(-\frac{(1-\beta) F V}{R T}\right)=0 .
\end{aligned}
$$

From (5) the adsorption isotherm is obtained:

$$
\frac{\theta}{1-\theta}=K_{1} a_{X^{-}} \exp \left(\frac{F V}{R T}\right)
$$

where $K_{1}=k_{1} / k_{-1}$. Hence, the degree of surface coverage is derived from equation (6):

$$
\theta=\frac{K_{1}^{\prime} \exp \left(\frac{F V}{R T}\right)}{1+K_{1}^{\prime} \exp \left(\frac{F V}{R T}\right)}=\frac{K_{1}^{\prime}}{\exp \left(-\frac{F V}{R T}\right)+K_{1}^{\prime}},
$$

with $K_{1}{ }^{\prime}=K_{1} \cdot a_{X^{-}}$. The expression for $\theta$ allows a different expression for the net faradaic current; it would be convenient to visualise the behaviour of the latter during the transient. Thus,

$$
i_{F}=k \frac{\mathrm{d} \theta}{\mathrm{d} t},
$$

where $k$ corresponds to the charge required to obtain a monolayer of adsorbed intermediates according to reaction (1). Equation (8) involves the rate of change of the degree of surface coverage, the latter depending on the applied potential, which is a function of time. The potential $V$ at time $t$ is expressed by:

$$
\boldsymbol{V}=\boldsymbol{V}_{\mathrm{i}}+v t-i_{\boldsymbol{F}} \boldsymbol{R}_{\mathrm{u}},
$$

where $V_{i}$ is the initial potential, in $V$; $v$ the potential sweep rate in $V / \mathrm{s}$ and $R_{\mu}$ is the ohmic drop across the cell, in $\Omega \times \mathrm{cm}^{2}$. Hence, the "true potential sweep rate," $v_{t}$, is given by:

$$
v_{t}=\frac{\mathrm{d} V}{\mathrm{~d} t}=v-\frac{\mathrm{d} i_{F}}{\mathrm{~d} t} R_{\mathrm{w}} .
$$

Combining equations (7), (8), (9) and $\left(9^{\prime}\right)$ one obtains:

$$
\begin{aligned}
\frac{\mathrm{d} \theta}{\mathrm{d} t}=\frac{K_{1}{ }^{\prime}}{\left[\exp \left(-\frac{F V}{R T}\right)+K_{1}{ }^{\prime}\right]^{2}} \frac{F}{R T} \\
\quad \times \exp \left[\left(-\frac{F V}{R T}\right)\left(v-\frac{\mathrm{di}_{F}}{\mathrm{~d} t} R_{\mathrm{u}}\right)\right] .
\end{aligned}
$$

Taking into account equation (10) with (8), the net faradaic current is:

$$
\begin{aligned}
i_{F}=\frac{k K_{1}^{\prime}}{\left[\exp \left(-\frac{F V}{R T}\right)+K_{1}^{\prime}\right]^{2}} \frac{F}{R T} \\
\quad \times \exp \left[\left(-\frac{F V}{R T}\right)\left(v-\frac{\mathrm{di}_{F}}{\mathrm{~d} t} R_{\mathrm{u}}\right)\right] .
\end{aligned}
$$

Equation (11) can be written as follows:

$$
\frac{\mathrm{d} i_{F}}{\mathrm{~d} t}=\frac{v}{R_{u}}-\frac{R T i_{F}\left[\exp \left(-\frac{F V}{R T}\right)+K_{1}^{\prime}\right]^{2}}{k K_{1}{ }^{\prime} F R_{u} \exp \left(-\frac{F V}{R T}\right)} .
$$

and taking into account (9):

$\frac{\mathrm{d} i_{F}}{\mathrm{~d} t}=\frac{v}{R_{u}}-\frac{R T i_{F}\left[\exp \left(-\frac{F\left(V_{i}+v t-i_{F} R_{u}\right.}{R T}\right)+K_{1}^{\prime}\right]^{2}}{k K_{1}^{\prime} F R_{\mathrm{u}}\left[\exp \left(-\frac{F\left(V_{i}+v t-i_{F} R_{u}\right)}{R T}\right)\right]}$.

This equation can be solved by applying a numerical method, such as the Runge-Kutta procedure, only if $R_{u} \neq 0$. The program was fed to a Hewlett-Packard calculator, the results being available from an X-Y plotter (Fig. 1) for a particular set of fixed conditions, as indicated in Table 1. Equation (13) implies the current peak potential shifts with potential as indicated in Fig. 2.

Equation (13) has, as a limiting case when $R_{u} \rightarrow 0$ $\left(R_{u} \neq 0\right)$, the kinetic equations derived by Srinivasan and Gileadi for the same type of process[7]. For the quasi-reversible case, when $\boldsymbol{R}_{\mu}=0$, the current peak height changes linearly with the potential sweep rate and the potential at the current peak is independent of the potential sweep rate.

\section{Reaction (1) under irreversible conditions}

Let us consider the possibility that this reaction is highly irreversible so that the reverse reaction can be neglected. Then the rate of the faradaic process is nearly equal to the rate of the forward reaction. Under these conditions, the applied potential is far from 
Table 1. Data used to calculate $E / I$ curves for reaction (1) under quasi-equilibrium conditions. $A=v / R_{u}{ }^{\prime} ; \quad Z=B / k K_{1} R_{u}^{\prime} ; \quad B=R T / \beta F ; \quad K_{1}=7.0685 \times 10^{-4} ; \quad k=13.56 \mathrm{mC} / \mathrm{cm}^{2} ; \quad t_{0}=$ $\left[\left(i_{F}\right)_{t=0}\right.$ Area $\left.R_{u} ; v\right] ; T=473^{\circ} \mathrm{K} ; B=41.3525 \mathrm{mV}$; Electrode Area: $1 \mathrm{~cm}^{2}$

\begin{tabular}{|c|c|c|c|c|c|c|}
\hline $\begin{array}{c}\boldsymbol{R}_{u}{ }^{\prime} \\
\boldsymbol{\Omega}\end{array}$ & $\begin{array}{c}v \\
\mathrm{mV} / \mathrm{s}\end{array}$ & $A$ & $Z$ & $\begin{array}{c}\Delta t \\
\mathrm{~s}\end{array}$ & $\begin{array}{c}\left(i_{F}\right)_{t=0} \\
\mathrm{~mA} / \mathrm{cm}^{2}\end{array}$ & $\begin{array}{c}t_{\mathbf{0}} \\
\mathrm{s}\end{array}$ \\
\hline 5 & 10 & $2 \cdot 00$ & $8.6293 \times 10^{2}$ & $2.5 \times 10^{-4}$ & $2.318 \times 10^{-3}$ & $1.159 \times 10^{-3}$ \\
\hline 50 & 10 & $5.0 \times 10^{-1}$ & $8.6293 \times 10^{1}$ & $2.5 \times 10^{-3}$ & $2.318 \times 10^{-3}$ & $1.159 \times 10^{-2}$ \\
\hline 100 & 10 & $10 \times 10^{-2}$ & $43146 \times 10^{1}$ & $5 \times 10^{-3}$ & $2.318 \times 10^{-3}$ & $2.318 \times 10^{-2}$ \\
\hline 200 & 10 & $5.0 \times 10^{-2}$ & $2.1573 \times 10^{1}$ & $1 \times 10^{-2}$ & $2.318 \times 10^{-3}$ & $4.636 \times 10^{-2}$ \\
\hline 300 & 10 & $3.33 \times 10^{-2}$ & $1.4382 \times 10^{1}$ & $1.5 \times 10^{-2}$ & $2.318 \times 10^{-3}$ & $6.954 \times 10^{-2}$ \\
\hline
\end{tabular}

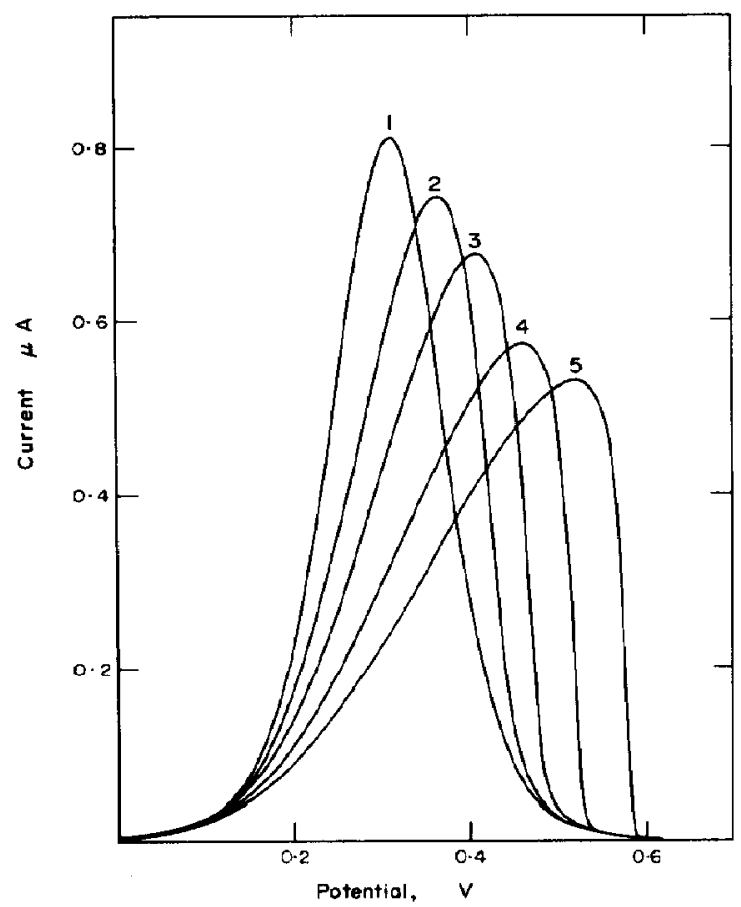

Fig. 1. Anodic current-potential curves obtained from equation (13), with data assembled in Table $1 . R_{u}^{\prime}=5 \Omega(1)$;

$50 \Omega(2) ; 100 \Omega(3) ; 200 \Omega(4) ; 300 \Omega(5) ; 500 \Omega(6)$.

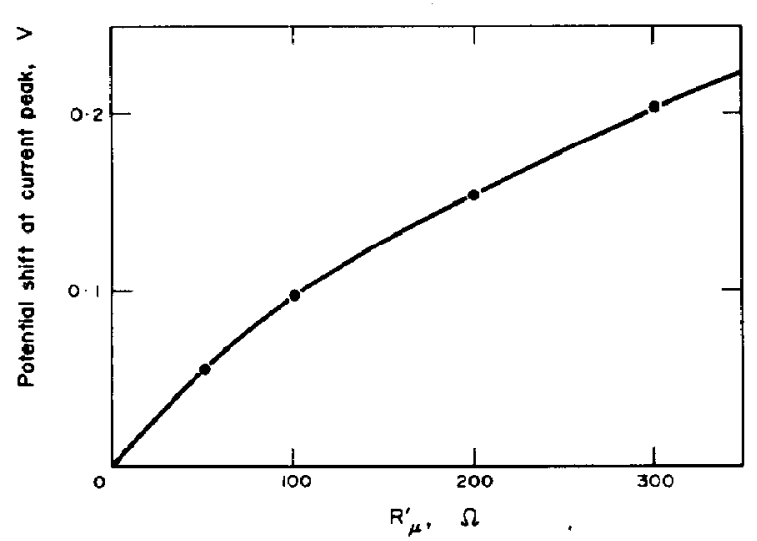

Fig. 2. Shift of the potential at peak current with ohmic resistance. The shifts are referred to the potential at current peak for $R_{u}{ }^{\prime}=0 \Omega$. the equilibrium potential and a Tafel relationship between $V$ and $i_{F}$ is fitted:

$$
i_{F}=k_{1} a_{X^{-}}(1-\theta) \exp \left(\frac{\beta F V}{R T}\right) .
$$

Taking into account (9), the rate equation in the differential form is:

$$
\begin{aligned}
\frac{\mathrm{d} i_{\boldsymbol{F}}}{\mathrm{d} t}= & k_{1}{ }^{\prime}(1-\theta) \frac{\mathrm{d}}{\mathrm{d} t}\left[\exp \left(\frac{\beta F}{R T}\left(V_{i}+v t-i_{F} R_{\mathrm{u}}\right)\right)\right] \\
& -k_{1} \exp \left(\frac{\beta F V}{R T}\right) \frac{\mathrm{d} \theta}{\mathrm{d} t} \\
= & k_{1}{ }^{\prime}(1-\theta) \exp \left(\frac{\beta F V}{R T}\right) \frac{\beta F}{R T}\left(v-\frac{\mathrm{d} i_{F}}{\mathrm{~d} t} R_{\mathrm{u}}\right) \\
& -\frac{k_{1}^{\prime}}{k} i_{F} \exp \left(\frac{\beta F V}{R T}\right)
\end{aligned}
$$

where $k_{1}^{\prime}=k_{1} a_{X^{-}}$. From (15) one obtains:

$$
1-\theta=\frac{\frac{\mathrm{d} i_{F}}{\mathrm{~d} t}+\frac{k_{1}{ }^{\prime}}{k} i_{F} \exp \left|\frac{\beta F V}{R T}\right|}{k_{1}{ }^{\prime} \exp \left[\frac{\beta F V}{R T}\right] \frac{\beta F V}{R T}\left(v-\frac{\mathrm{d} i_{F}}{\mathrm{~d} t} R_{\mathrm{u}}\right)} .
$$

Considering equations (14) and (17), the net faradaic current is given by the expression:

$i_{F}=\left[\frac{\mathrm{d} i_{F}}{\mathrm{~d} t}+\frac{k_{1}^{\prime}}{k} i_{F} \exp \left(\frac{\beta F V}{R T}\right)\right]\left[\left(v-\frac{\mathrm{d} i_{F}}{\mathrm{~d} t} R_{u}\right) \frac{\beta F}{R T}\right]^{-1}$.

From (18) the explicit differential expression is:

$$
\frac{\mathrm{d} i_{F}}{\mathrm{~d} t}=\frac{i_{F} \frac{\beta F}{R T} v-\frac{k_{1}{ }^{\prime}}{k} \exp \left(\frac{\beta F V}{R T}\right) i_{F}}{1+i_{F} R_{\mathrm{w}} \frac{\beta F}{R T}} .
$$

This equation, replacing the expression for $V$, from (9), becomes:

$$
\frac{\mathrm{d} i_{F}}{\mathrm{~d} t}=\frac{\frac{i_{F} v \beta F}{R T}-\frac{i_{F} k_{1}{ }^{\prime}}{k} \exp \left[\frac{\beta F}{R T}\left(V_{i}+v t-i_{F} R_{u}\right)\right]}{1+i_{F} R_{u} \frac{\beta F}{R T}} .
$$


To solve equation (20), the numerical procedure for the evaluation of equation (13) was again used. Solutions for any value of $R_{u}$, including $R_{u}=0$, can be obtained. The kinetic parameters used are assembled in Table 2; they correspond to parathiocyanogen formation on platinum at $190^{\circ} \mathrm{C}$ in molten potassium

Table 2. Data used to calculate $\mathbf{E} / \mathbf{I}$ curves for reaction (1) under irreversible conditions. $M=(R T / \beta F) \times$ $\left(k_{1} / k v\right) ; \quad k_{1}=1.532 \times 10^{-15} \mathrm{~A} / \mathrm{cm}^{2}$; $k=28.98 \mathrm{mC} / \mathrm{cm}^{2}$; Electrode area: $0.102 \mathrm{~cm}^{2}$. Numerical data taken from ref. [18].

$t_{0}=\left[(i)_{t=0}\right.$ Area $\left.R_{u}^{\prime} / v\right] ; \quad(i)_{t=0}=k_{1} ;$ $v=15 \mathrm{mV} / \mathrm{s} ; \mathrm{M}=7.01 \times 10^{-14}$

\begin{tabular}{rcc}
\hline $\begin{array}{c}R_{u}{ }^{\prime} \\
\Omega\end{array}$ & $\begin{array}{c}\Delta t \\
\mathrm{~s}\end{array}$ & $\begin{array}{c}t_{\mathrm{u}} \\
\mathrm{s}\end{array}$ \\
\hline 0 & $5 \times 10^{-2}$ & 0 \\
10 & $2 \times 10^{-2}$ & $1.042 \times 10^{-13}$ \\
56 & $2 \times 10^{-2}$ & $5.834 \times 10^{-13}$ \\
100 & $2 \times 10^{-2}$ & $1.0213 \times 10^{-12}$ \\
150 & $1 \times 10^{-2}$ & $1.532 \times 10^{-12}$ \\
220 & $1 \times 10^{-2}$ & $2.2469 \times 10^{-12}$ \\
330 & $1 \times 10^{-3}$ & $3.4725 \times 10^{-12}$ \\
470 & $1 \times 10^{-3}$ & $4.9024 \times 10^{-12}$ \\
1000 & $1 \times 10^{-3}$ & $1.0213 \times 10^{-11}$ \\
2000 & $1 \times 10^{-3}$ & $2.0427 \times 10^{-11}$ \\
\hline
\end{tabular}

thiocyanate. Results are shown in Fig. 3, as given by the X-Y plotter.

If the current maximum is $i_{F, \max }$ and the corresponding potential is $V_{\max }$, the condition $\left(\mathrm{d} i_{F} / \mathrm{d} t\right)=0$ implies:

$$
\frac{\beta F v}{R T}=\frac{k_{1}{ }^{\prime}}{k} \exp \left[\frac{\beta F V_{\max }}{R T}\right] \text {. }
$$

Therefore:

$$
V_{\max }=\frac{R T}{\beta F} \ln \frac{\beta F}{R T} \frac{k}{k_{\mathrm{l}}{ }^{\prime}}+\frac{R T}{\beta F} \ln v+I_{\max } R_{\mathrm{u}}^{\prime}
$$

According to (22) the potential at the peak current, at a constant $R_{u}{ }^{\prime}$ value, increases linearly with the logarithm of the potential sweep rate (Fig. 4). $R_{u}{ }^{\prime}$ is the ohmic drop across the cell in $\Omega$. The ohmic drop appears as a term which depends on $v$, as the maximum current does. Consequently a $V_{\max } v s \ln v$ plot does not give a straight line if the quantity of $R_{u}{ }^{\prime}$ is appreciable. Equation (22), when $R_{u}{ }^{\prime}=0$, produces the equation derived by Gileadi and Srinivasan[7].

\section{A second order electrochemical process under irreversible conditions}

Let us consider a second order electrochemical reaction under activation control represented by:

$$
2 M X+n e \rightleftharpoons X_{2}{ }^{n-}+2 M
$$

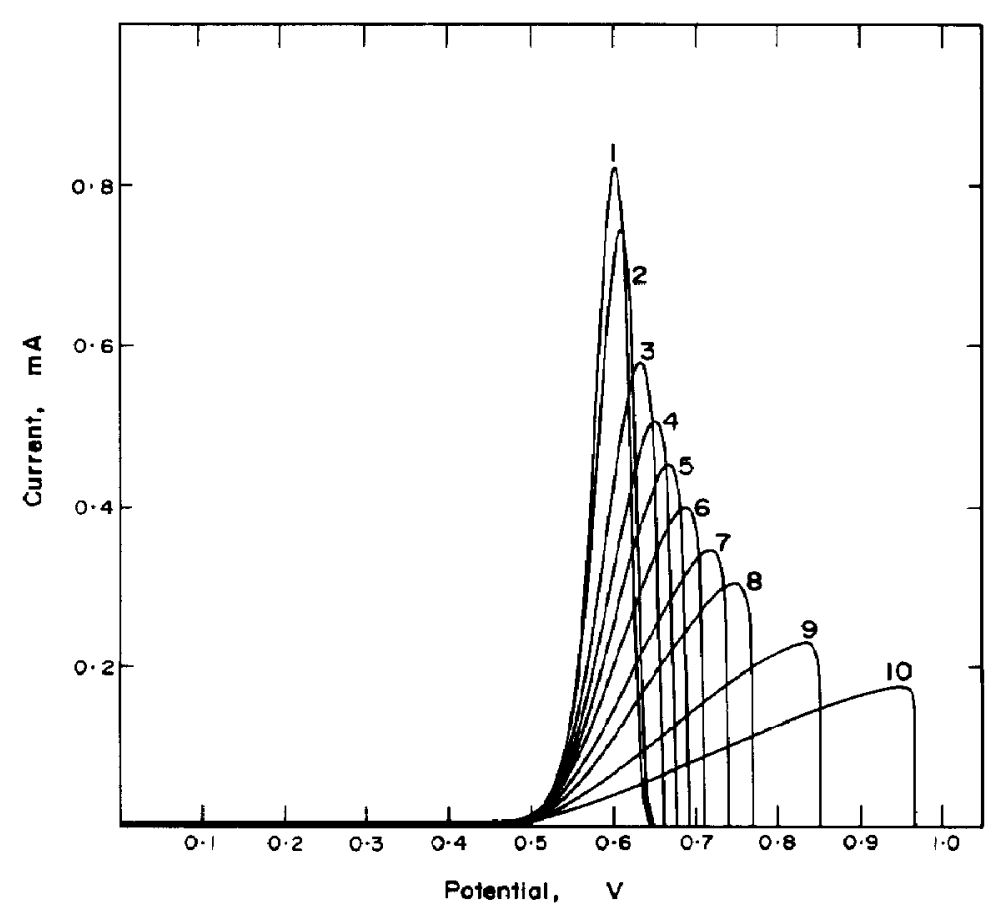

Fig. 3. Anodic current-potential curves obtained from equation (20), with data assembled in Table 2. $R_{u}^{\prime}=0 \Omega(1) ; 10 \Omega(2) ; 56 \Omega(3) ; 100 \Omega(4) ; 150 \Omega(5) ; 220 \Omega(6) ; 330 \Omega(7) ; 470 \Omega(8) ; 1000 \Omega(9) ;$ 
This reaction implies the desorption and clcctrochemical reduction of the species $X$, which is initially adsorbed on the electrode surface, the step involving the simultaneous transfer of $n$ charges. As in case (2). at potentials far from equilibrium the rate of the faradaic process in the forward direction under stationary conditions is:

$$
i_{F}=-k_{2} \theta^{2} \exp \left(-\frac{\alpha_{2} n F V}{R T}\right) .
$$

Taking into account the equation for the applied potential:

$$
V=V_{i}-v t+i_{F} R_{u}
$$

one obtains:

$$
i_{F}=-k_{2} \theta^{2} \exp \left[\frac{\left(V_{i}+v t-i_{F} R_{u}\right) \alpha_{2} n F}{R T}\right]
$$

and differentiation yields:

$$
\begin{aligned}
\frac{\mathrm{d} i_{F}}{\mathrm{~d} t} & \\
= & -k_{2} 2 \theta \frac{\mathrm{d} \theta}{\mathrm{d} t} \exp \left(\frac{\left(V_{i}+v t-i_{F} R_{u}\right) \alpha_{2} n F}{R T}\right) \\
& -\frac{k_{2} \theta^{2} \alpha_{2} n F}{R T}\left(v-\frac{\mathrm{d} i_{F}}{\mathrm{~d} t} R_{u}\right) \\
& \times \exp \left[\frac{\left(V_{i}+v t-i_{F} R_{\mathrm{u}}\right) \alpha_{2} n F}{R T}\right] \\
= & -2 \frac{k_{2}{ }^{1 / 2}}{k} i_{F}{ }^{3 / 2} \exp \left[\frac{\left(V_{i}+v t-i_{F} R_{\mathrm{u}}\right) \alpha_{2} n F}{2 R T}\right] \\
& +\frac{i_{F} \alpha_{2} n F}{R T}\left(v-\frac{\mathrm{d} i_{F}}{\mathrm{~d} t} R_{\mathrm{u}}\right) \\
= & -\left\{\frac{2 k_{2}{ }^{1 / 2}}{k} i_{F}^{3 / 2} \exp \left[\frac{\left(V_{i}+v t-i_{F} R_{\mathrm{u}}\right) \alpha_{2} n F}{2 R T}\right]\right. \\
& \left.-\frac{i_{F} v \alpha_{2} n F}{R T}\right\}\left(1+\frac{i_{F} R_{\mathrm{u}} \alpha_{2} n F}{R T}\right)^{-1} \cdot
\end{aligned}
$$

The condition for the current peak, $\left(\mathrm{d} i_{F} / \mathrm{d} t\right)=0$, is fulfilled when:

$$
\frac{2 k_{2}^{1 / 2}}{k} i_{\max }^{1 / 2} \exp \left[\frac{\left(V_{i}+v t-i_{\max } R_{u}\right) \alpha_{2} n F}{2 R T}\right]=\frac{\alpha_{2} n F v}{R T} .
$$

Therefore:

$$
\begin{aligned}
\left(V_{i}+v t\right)_{\max } & -i_{\max } R_{u} \\
& =\frac{2 R T}{\alpha_{2} n F} \ln \frac{\alpha_{2} n F v}{R T k_{2}{ }^{1 / 2}}+\frac{2 R T}{\alpha_{2} n F} \ln \frac{k}{2 i_{\max }^{1 / 2}},
\end{aligned}
$$

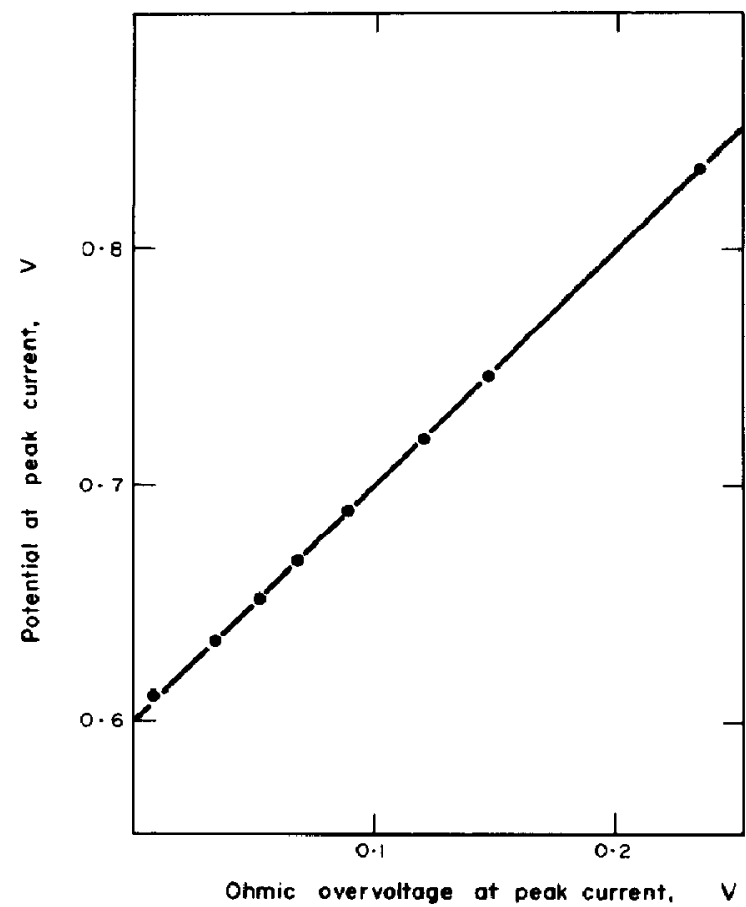

Fig. 4. Dependence of the potential at peak current on ohmic overvoltage according to (22). Data taken from Fig. 3.

which can be rearranged as follows:

$$
\begin{aligned}
V_{\max }=i_{\max } R_{\mu}+ & \frac{2 R T}{\alpha_{2} n F} \ln \frac{\alpha_{2} n F}{R T} v \\
& \quad-\frac{R T}{\alpha_{2} n F} \ln k_{2}+\frac{2 R T}{\alpha_{2} n F} \ln \frac{k}{2 i_{\max }^{1 / 2}} .
\end{aligned}
$$

Equation (30) implies a relationship between $V_{\max }$ and $v$ which depends on $i_{\max }$ through a linear term and a logarithmic term, the latter depending on the order of the electrode reaction. When reaction (30) is compared to the one deduced in the absence of the ohmic polarisation term, we obtain:

$$
\begin{aligned}
V_{\max }=\left(V_{\max }\right)_{R_{k}=0} & +\frac{R T}{\alpha_{2} n F} \ln \frac{\alpha_{2} n F}{R T} \frac{v}{q_{0}} \\
& +\frac{R T}{\alpha_{2} n F} \ln \frac{k^{2}}{4 n F i_{\max }}+i_{\max } R_{u},
\end{aligned}
$$

where, $q_{0}$ is the number of mole $/ \mathrm{cm}^{2}$ initially placed at the electrode surface. If $k_{2}$ is the second order rate constant, expressed as a current density, the following relationship is satisfied:

$$
k_{2}=n F q_{0}{ }^{2} k_{1 \mathrm{II}}
$$

$k_{\mathrm{fI}}$ being the second order rate constant in $\mathrm{cm}^{2} / \mathrm{s}$.mole.

A set of theoretical voltammetric E/I curves, computed from (27), for a cathodic second order desorption plus electron transfer process, obtained for different 


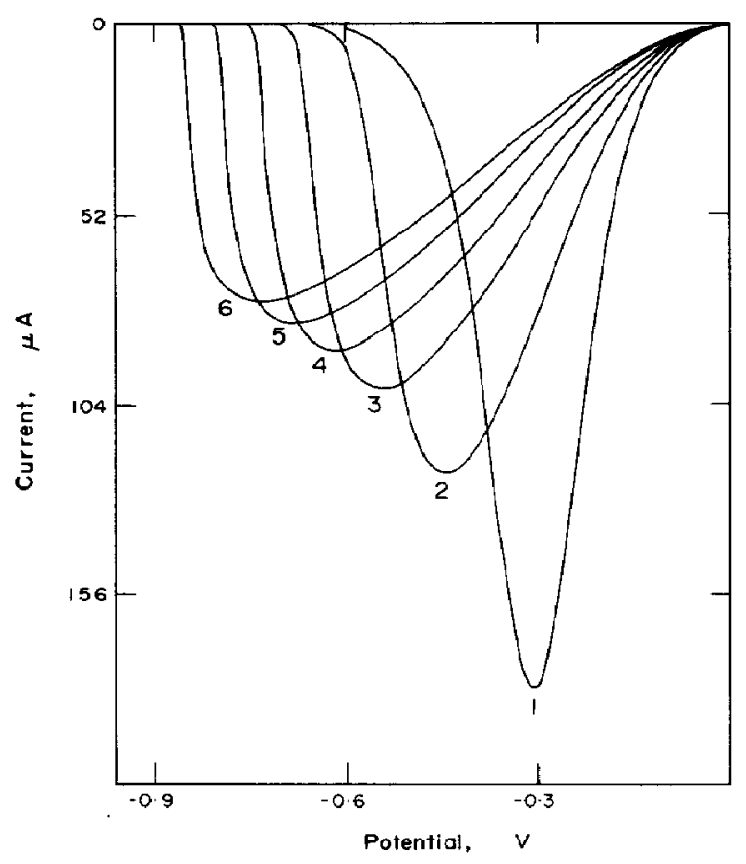

Fig. 5. Experimental cathodic current-potential curves for the electrochemical reduction of platinum oxide in $1 \mathrm{~N}$ sulphuric acid at room temperature, at $300 \mathrm{mV} / \mathrm{s} . K_{u}{ }^{\prime}=$ $0 \Omega(1) ; 1947 \Omega(2) ; 4000 \Omega(3) ; 6312 \Omega(4) ; 7937 \Omega(5)$; and $10,000 \Omega(6)$. See Tables 3 and 4 .

Table 3. Data used for computation of the voltammetric E/I curves with equation (27) for the reduction of platinum oxide in $1 N$ sulphuric acid at $294 \cdot 2^{\circ} \mathrm{K}$

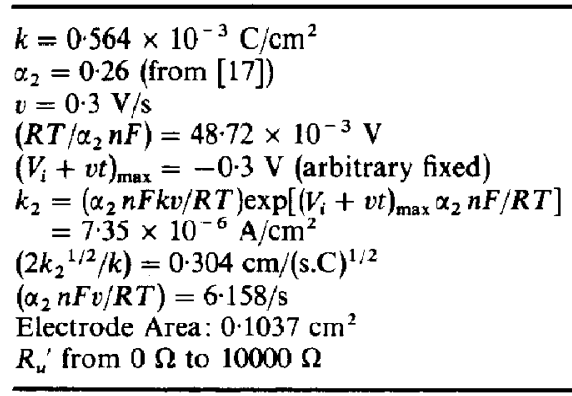

values of $R_{u}^{\prime}$ are showed in Fig. 5. The values of the constants used are assembled in Table 3. The $\alpha_{2} n$ value for the reduction of platinum oxide was taken from the paper by Ohashi, Sasaki and Nagaura[17].

In order to test the validity of equation (30), it was necessary to record new current-potential curves by cyclic voltammetry for the platinum oxide reduction in $1 \mathrm{~N} \mathrm{H}_{2} \mathrm{SO}_{4}$ at room temperature as made earlier by the authors mentioned above[17]. For this purpose, a constant amount of oxide formed on a clean platinum electrode by an anodic voltammetric sweep under conditions of $R_{u}^{\prime} \rightarrow 0$, was reduced by a cathodic voltammetric sweep. The cathodic reaction occurred by inserting in the circuit, by means of a rapid switching operation, a resistor of prefixed value. The potential sweep covered the voltage range from -0.910 to $+0.836 \mathrm{~V}$. Results are assembled in Table 4 , as well as the difference, $\Delta$, between the experimental and the theoretical values.

\section{CONCLUSIONS}

The mathematical analysis of current-potential transients obtained by the application of the linear potential sweep method for a simple first order adsorption step and rate controlling involving charge transfer under Langmuir isotherm, including the effect of the ohmic resistance, yields two differential equations which correspond to the following limiting cases: (i) quasi-equilibrium conditions and (ii) Tafel approximation. The solution of both equations gives the quantitative effect of the uncompensated ohmic cell resistance on the voltagrams. The errors involved in the calculations are the following: (i) for the quasireversible case, for $R_{u}{ }^{\prime}=300 \Omega$ and the conditions indicated in Table 1, the error in the current peak is $6 \times 10^{-5} \mathrm{~mA}$. (ii) for the irreversible case, for $R_{u}{ }^{\prime}=$ $10 \Omega$ and conditions indicated in Table 2 , the error in the current peak amounts to $7.83 \times 10^{-9} \mathrm{~mA}$.

The ohmic drop correction depends also on the order of the electrochemical reaction, as is clearly demonstrated with quantitative data obtained for the electrochemical reduction of platinum oxide in sulphuric acid at room temperature.

In conclusion the uncompensated ohmic effect, together with the double layer capacity effect, may

Table 4. Experimental and theoretical values of $\left(V_{i}+v t\right)_{\max }$ at different $R_{u}$ for the second order electrochemical reduction of platinum oxide

\begin{tabular}{|c|c|c|c|c|c|c|c|}
\hline $\begin{array}{l}\boldsymbol{R}_{u}{ }^{\prime} \\
\boldsymbol{\Omega}\end{array}$ & $\begin{array}{l}I_{\max } \\
\mu \mathrm{A}\end{array}$ & $\begin{array}{c}{\left[\left(V_{i}+v t\right)_{R_{u}^{\prime}=0}\right.} \\
\left.-\left(V_{i}+v t\right)_{R_{i}=R_{i}}\right]_{\exp } \\
\mathrm{mV}\end{array}$ & $\begin{array}{c}I_{\max } R_{u}^{\prime} \\
\mathrm{mV}\end{array}$ & $\frac{R T}{\alpha_{2} n F}$ & $\ln _{\mathrm{mV}} \frac{\alpha_{2} n F k v}{4 R T I_{\max }}$ & $\begin{array}{c}{\left[\left(V_{i}+v t\right)_{R_{u}^{\prime}=0^{-}}\right.} \\
\left.-\left(V_{i}+v t\right)_{R_{u}^{\prime}=R_{u}^{\prime}}\right]_{\text {heor }} \\
\mathrm{mV}\end{array}$ & $\begin{array}{l}|\Delta| \\
m V\end{array}$ \\
\hline 0 & $90 \pm 8$ & 0 & 0 & & - & 0 & 0 \\
\hline 1947 & 64 & $148 \pm 10$ & 124.6 & & 16.63 & $141 \cdot 2$ & $|+6 \cdot 8|<10$ \\
\hline 4000 & 57 & $259 \pm 10$ & $228 \cdot 0$ & & $22 \cdot 25$ & $250 \cdot 3$ & $|+8.4|<10$ \\
\hline 6312 & 53 & $370 \pm 10$ & 334.5 & & 25.96 & $360 \cdot 5$ & $|+9.5|<10$ \\
\hline 7937 & 46 & $402 \pm 10$ & $365 \cdot 1$ & & $32 \cdot 86$ & 398.0 & $|+4 \cdot 0|<10$ \\
\hline 10000 & 41 & $438 \pm 10$ & $410 \cdot 0$ & & $38 \cdot 30$ & $448 \cdot 3$ & $|-10 \cdot 3| \approx 10$ \\
\hline
\end{tabular}


produce a large distortion in the voltammetric currentpotential curves so that kinetic data derived from peak currents and from the whole curve can be significantly erroneous. This effect is particularly notorious when large potential sweep rates are employed and is important for the case of Nernstian charge transfer process and for an electrochemical process, which are controlled by a charge transfer step. For the latter it can also be predicted that the influence of the uncompensated ohmic resistance term decreases when passing from a Langmuir to a Temkin isotherm condition and in this case it also decreases when the Temkin factor, $f$, increases for then the current at the peak becomes smaller[18].

Acknowledgement-This work is part of the research program of INIFTA, sponsored by the University of La Plata, the Consejo Nacional de Investigaciones Científicas y Técnicas and the Comisión de Investigaciones Cientificas de la Provincia de Buenos Aires.

\section{REFERENCES}

1. A. Sevcik, Colln. Czech. chem. Commun. 13, 349 (1948).

2. P. Delahay, New Instrumental Methods in Electrochemistry, Ch. 6, Interscience, New York (1954).
3. H. Matsuda and Y. Ayabe, Z. Elektruchem. 59, 494 (1955).

4. R. S. Nicholson and 1. Shain, Analyt. Chem. 36, 706 (1964).

5. R. S. Nicholson, Analyt. Chem. 37, 667 (1965).

6. W. T. DeVr.es and E. Van Dalen, J. electroanal. Chem. 10, 183 (1965).

7. S. Srinivasan and E. Gileadi, Electrochim. Acta 11, 321 (1966).

8. A. G. Voloshin, Elektrokhimiya 3, 924 (1967).

9. S. Roffia, Ric. Sci. 38, 1257 (1968).

10. P. Stonehart, Electrochim. Acta 13, 1789 (1968).

11. S. Roffia and M. Lavacchieli, J. electroanal. Chem. 22, 117 (1969).

12. C. P. Andrieux, L. Nadjo and J. M. Savéant, $J$. electroanal. Chem. 26, 147 (1970).

13. J. Clavilier, C. hebd. Séanc. Acad. Sci., Paris 270, 1698 (1970).

14. E. Laviron, Bull. Soc. chim. Fr. 3717 (1967); Electrochim. Acta 16, 409 (1971).

15. J. C. Imbeaux and J. M. Savéant, $J$. electroanal. Chem. 28, 325 (1970); 31, 183 (1971).

16. A. J. Arvia, A. J. Calandra and M. E. Martins, Electrochim. Acta 17, 741 (1972).

17. K. Ohashi, K. Sasaki and S. Nagaura, Bull. chem. Soc. Japan 39, 2066 (1966).

18. J. M. Hale and R. Greef, Electrochim. Acta 12, 1409 (1967). 